\title{
On the Differentiability of Vector Valued Additive Set Functions
}

\author{
Mangatiana A. Robdera, Dintle Kagiso \\ Department of Mathematics, University of Botswana, Gaborone, Botswana \\ Email: robdera@yahoo.com,dintlek.kagiso@gmail.com
}

Received October 7, 2013; revised November 8, 2013; accepted November 15, 2013

Copyright (C) 2013 Mangatiana A. Robdera, Dintle Kagiso. This is an open access article distributed under the Creative Commons Attribution License, which permits unrestricted use, distribution, and reproduction in any medium, provided the original work is properly cited.

\begin{abstract}
The Lebesgue-Nikodým Theorem states that for a Lebesgue measure $\lambda: \Sigma \subset 2^{\Omega} \rightarrow[0, \infty)$, an additive set function $F: \Sigma \rightarrow \mathbb{R}$ which is $\lambda$-absolutely continuous is the integral of a Lebegsue integrable a measurable function $f: \Omega \rightarrow \mathbb{R}$; that is, for all measurable sets $A, F(A)=\int_{A} f \mathrm{~d} \lambda$. Such a property is not shared by vector valued set functions. We introduce a suitable definition of the integral that will extend the above property to the vector valued case in its full generality. We also discuss a further extension of the Fundamental Theorem of Calculus for additive set functions with values in an infinite dimensional normed space.
\end{abstract}

Keywords: Vector Valued Additive Set Function; Lebesgue-Radon-Nikodým Theorem; Fundamental Theorem of Calculus

\section{Introduction}

This note can be considered as a continuation of the work started by the first author in [1]. Throughout the paper $\Sigma \subset 2^{\Omega}$ is a $\sigma$-ring of subsets of a nonempty set $\Omega$. A vector measure is a countably additive set function $\mu$ defined on $\Sigma$ taking values in a Banach space $X$ and such that $\mu(\varnothing)=0$. We denote by $M(\Sigma, X)$ the set of all $X$-valued vector measures defined on $\Sigma$.

Let $\lambda: \Sigma \rightarrow[0, \infty)$ be a nonnegative scalar measure. If a function $f: \Omega \rightarrow X$ is $\lambda$-Lebesgue-Bochner integrable, then it is easily seen that the function $F: \Sigma \rightarrow X$ defined by $F(A)=\int_{A} f \mathrm{~d} \lambda$ is a vector measure. It readily follows from the property of the Lebesgue-Bochner integral that $F(A)=0$ whenever $\lambda(A)=0$. Such a property is expressed by saying that $F$ is absolutely continuous with respect to $\lambda$.

Definition 1. A set function $F: \Sigma \rightarrow X$ is said to be $\lambda$-Lebesgue-Bochner differentiable if there exists a $\lambda$ Lebesgue-Bochner integrable function $f: \Omega \rightarrow \mathbb{R}$, such that for every $A \in \Sigma, \quad F(A)=\int_{A} f \mathrm{~d} \lambda$.

Such a function $f$ is called the $\lambda$-Radon-Nikodým derivative of $F$. It easily follows from the property of the Lebesgue-Bochner integral that if $f$ is a $\lambda$-Ra-
don-Nikodým derivative of $F$ then so is any function $g$ such that $\lambda(\{x \in \Omega: f(x)=g(x)\})=0$. A representative of the class of such functions is denoted by $\frac{\mathrm{d} F}{\mathrm{~d} \lambda}$.

The classical Lebesgue-Nikodým theorem states that for an additive real valued set function (not necessarily countably additive) $F: \Sigma \rightarrow \mathbb{R}, \lambda$-absolutely continuity implies $\lambda$-Lebesgue differentiability (see for example [2]). The analogue of the Lebesgue-Nikodým theorem does not extend to the vector valued case in general. For instance, it is a well known fact that the $L^{1}([0,1])$-valued vector measure defined by $\mu(A)=1_{A}$ for every Borel subset $A$ of $[0,1]$, is absolutely continuous with respect to the Lebesgue measure $\lambda$ on $[0,1]$ but fails to be $\lambda$ Lebesgue-Bochner differentiable (see for example [3]).

In this note, we reactualize the approach to the definition of the integral first introduced in [1] in order to obtain a Lebesgue-Nikodým type theorem on the differentiability of Banach space valued additive set function.

The exposition will be organized as follows. In Section 2 , we introduce a new approach to integration theory that does not require the elaborate machinery of Lebesgue measure theory, and at the same time significantly sim- 
plifies the approach to gauge integral. In Section 3, we shall see that the space of classes of integrable functions (in the sense of the definition of integrability in Section 2) can be naturally given a Banach space structure. In Section 4 , we state and prove our main result which can be seen as an extended vector valued version of the Lebesgue-Nikodým theorem. The fifth section is devoted to some extension of the Fundamental Theorem of Calculus.

\section{Extended Notion of Integral}

We begin by recalling the definition of limit in its most general form, that is, the Moore-Smith limit, also known as the net limit.

A nonempty set $D$ is said to be directed by a binary relation $\succ$, if $\succ$ has the following properties:

1) for $x, y, z \in D$ if $x \succ y$ and $y \succ z$, then $x \succ z$ (transitivity);

2) for $x, y \in D$, there exists $z \in D$ such that $z \succ x$ and $z \succ y$ (upper bound property).

Given a set $X$, a net of elements of $X$ is an $X$-valued function defined on a directed set $(D, \succ)$. The notion of convergence can then be defined whenever the set $X$ is a metric space, with distance function $d$.

A net $f:(D, \succ) \rightarrow(X, d)$ is said to be convergent if there exists an element $x \in X$ denoted by $x:=\lim _{(D, y)} f$

such that for every $\varepsilon>0$, there exists $\omega_{0} \in D$ such that for every $\omega \succ \omega_{0}, \quad \mathrm{~d}(f(\omega), x)<\varepsilon$.

Ordinary sequences $n \mapsto a_{n}$, in which $D=\mathbb{N}$ directed by $>$, constitute a special case of nets. The net limit takes over all the essential parts of the theory of limits of sequences; to name a few: the uniqueness of limit, the algebraic properties of limits, the monotone convergence property of limits, the Cauchy criterion, and so on. For more details on net limits, we refer the reader to [4].

Next we introduce the notion of size-function that will generalize the usual notion of measure in integral theory. In the following, $\Omega$ is a nonempty set. The power set of $\Omega$, that is, the set of all subsets of $\Omega$ will be denoted by $2^{\Omega}$.

Definition 2. Let $\Sigma \subset 2^{\Omega}$. By a size-function, we mean a set-function $\eta: \Sigma \rightarrow[0,+\infty]$ that satisfies the following conditions:

- $\eta(\varnothing)=0$

- $\eta(A) \leq \eta(B)$ whenever $A \subset B$ in $\Sigma$ (monotonicity);

- $\eta\left(\bigcup_{n \in \mathbb{N}} A_{n}\right) \leq \sum_{n \in \mathbb{N}} \eta\left(A_{n}\right)$ for every sequence $n \mapsto A_{n}$ in $\Sigma$ such that $\bigcup_{n \in \mathbb{N}} A_{n} \in \Sigma$ (countable subadditivity).

Obviously, any measure defined on a $\sigma$-ring is a size-function. The length function defined on the $\sigma$ - ring generated by the bounded intervals in $\mathbb{R}$ is another example of size function.

It is clear from the definition that an outer-measure is a size-function defined on $\Sigma=2^{\Omega}$. In fact, an arbitrary size-function $\eta: \Sigma \rightarrow[0,+\infty]$ defined on a $\sigma$-ring $\Sigma$ can be extended to an outer-measure defined on the whole of the power set $2^{\Omega}$ as follows.

Proposition 3. Let $\eta: \Sigma \rightarrow[0,+\infty]$ be a size-function. For every $A \subset \Omega$, define

$$
\eta^{*}(A)=\inf \left\{\sum_{n=1}^{\infty} \eta\left(I_{n}\right): A \subset \bigcup_{n} I_{n}, I_{n} \in \Sigma\right\} .
$$

Then $\eta^{*}$ is an outer-measure such that $\eta^{*}(E)=\eta(E)$ for every $E \in \Sigma$.

Definition 4. Let $\Sigma$ be an arbitrary subset of $2^{\Omega}$, and let $A \in \Sigma . A \Sigma$-subpartition $P$ of $A$ is any finite collection

$$
\left\{I_{i} ; I_{i} \subset A, I_{i} \in \Sigma, i=1,2, \cdots, n\right\}
$$

with the following properties:

1) $\eta\left(I_{i}\right)<\infty$ for all $i \in\{1, \cdots, n\} \quad I_{i} \subset A$,

2) $I_{i} \in \Sigma$, and $I_{i} \cap I_{j}=\varnothing$ whenever $i \neq j$.

We denote by $\sqcup P$ the subset of $A$ obtained by taking the union of all elements of $P$. A $\Sigma$-subpartition $P=\left\{I_{i}: i=1, \cdots, n\right\}$ is said to be tagged if a point $t_{i} \in I_{i}$ is chosen for each $i \in\{1, \cdots, n\}$. We write $P:=\left\{\left(I_{i}, t_{i}\right): i \in\{1, \cdots, n\}\right\}$ if we wish to specify the tagging points. We denote by $\Pi(A, \Sigma)$ the collection of all tagged $\Sigma$-subpartitions of the set $A$. The mesh or the norm of $P \in \Pi(A, \Sigma)$ is defined to be

$$
\|P\|=\max \left\{\eta\left(I_{i}\right): I_{i} \in P\right\} .
$$

Definition 5. If $P, Q \in \Pi(A, \Sigma)$, we say that $Q$ is a refinement of $P$ and we write $Q \succ P$ if $\|Q\| \leq\|P\|$ and $\sqcup P \subset \sqcup Q$.

It is readily seen that such a relation does not depend on the tagging points. It is also easy to see that the relation $\succ$ is transitive on $\Pi(A, \Sigma)$. If $P, Q \in \Pi(A, \Sigma)$, we denote by

$$
P \vee Q:=\{I \backslash J, I \cap J, J \backslash I: I \in P, J \in Q\} .
$$

Clearly, $P \vee Q \in \Pi(A, \Sigma), P \vee Q \succ P$ and $P \vee Q \succ Q$. Thus the relation $\succ$ has the upper bound property on $\Pi(A, \Sigma)$. We then infer that the set $\Pi(A, \Sigma)$ is directed by the binary relation $\succ$.

In what follows, $X$ will denote either a real or a complex normed vector space. Given a function $f: \Omega \rightarrow X$, and a tagged $\Sigma$-subpartition

$$
P=\left\{\left(I_{i}, t_{i}\right): i \in\{1, \cdots, n\}\right\} \in \Pi(A, \Sigma),
$$

we define the $(\Sigma, \eta)$-Riemann sum of $f$ on $P$ to be the vector 


$$
\eta_{\Sigma, f}(P)=\sum_{i=1}^{n} \eta\left(I_{i}\right) f\left(t_{i}\right) .
$$

Thus the function $P \mapsto \eta_{\Sigma, f}(P)$ is an $X$-valued net defined on the directed set $(\Pi(A, \Sigma), \succ)$. For convenience, we are going to denote $\int_{A} f \mathrm{~d} \eta_{\Sigma}:=\lim _{(\Pi(A, \Sigma), \succ} \eta_{\Sigma, f}$ whether or not the net limit exists.

We are now ready to give the formal definition of the resulting integral.

Definition 6. We say that a function $f: \Omega \rightarrow X$ is $(\Sigma, \eta)$-integrable over a set $A \in \Sigma$, if $\int_{A} f \mathrm{~d} \eta_{\Sigma}$ represents a vector in $X$. The vector $\int_{A} f \mathrm{~d} \eta_{\Sigma}$ is then called the $(\Sigma, \eta)$-integral of $f$ over the set $A$.

In other words, $f: \Omega \rightarrow X$ is $(\Sigma, \eta)$-integrable over the set $A$ with $(\Sigma, \eta)$-integral $\int_{A} f \mathrm{~d} \eta_{\Sigma}$ if for every $\varepsilon>0$, there exists $P_{0} \in \Pi(A, \Sigma)$, such that for every $P \in \Pi(A, \Sigma), \quad P \succ P_{0} \quad$ we have

$$
\left\|\int_{A} f \mathrm{~d} \eta_{\Sigma}-\eta_{\Sigma, f}(P)\right\|<\varepsilon .
$$

We shall denote by $\mathcal{I}(A, X,(\Sigma, \eta))$ the set of all $(\Sigma, \eta)$-integrable functions over the set $A$.

Many classical properties of the integral follow immediately from the properties of net limits and therefore their proofs are obtained at no extra cost. To name a few, we have the uniqueness of the integral, the linearity of the integral operator, the Cauchy criterion for integrability, and so on.

It is also important to realize that no notion of measurability nor notion of gauge is postulated in the above definition.

We finish this section by noticing that if $\Sigma_{1} \subset \Sigma_{2}$ in $2^{\Omega}$, then $\Pi\left(A, \Sigma_{1}\right) \subset \Pi\left(A, \Sigma_{2}\right)$. Hence, we have the following proposition stating the relationship between $\left(\Sigma_{1}, \eta\right)$-integrability and $\left(\Sigma_{2}, \eta\right)$-integrability.

Proposition 7. Assume that $\Sigma_{1} \subset \Sigma_{2}$ in $2^{\Omega}$. Then for every $A \in 2^{\Omega}$,

$$
\mathcal{I}\left(A, X,\left(\Sigma_{1}, \eta\right)\right) \subset \mathcal{I}\left(A, X,\left(\Sigma_{2}, \eta\right)\right)
$$

and

$$
\int_{A} f \mathrm{~d} \eta_{\Sigma_{1}}=\int_{A} f \mathrm{~d} \eta_{\Sigma_{2}} \text { for } f \in \mathcal{I}\left(A, X,\left(\Sigma_{1}, \eta\right)\right) .
$$

\section{Spaces of Integrable Functions}

In this section, we show that the space $\mathcal{I}(A, X,(\Sigma, \eta))$ can be given a structure of complete seminormed space. We fix a (real or complex) normed vector space $X$. In view of Proposition 3 and Proposition 7, we can always regard integrability only with respect to a size-function $\eta$ defined on the whole of $2^{\Omega}$ and we shall write $\int_{A} f \mathrm{~d} \eta$ in stead of $\int_{A} f \mathrm{~d} \eta_{\Sigma}, \Pi(A)$ in stead of $\Pi\left(A, 2^{\Omega}\right)$, and
$\mathcal{I}(A, X, \eta)$ in stead of $\mathcal{I}\left(A, X,\left(2^{\Omega}, \eta\right)\right)$.

Clearly, a Riemann integrable (resp. Lebesgue integrable) function can be regarded as an integrable function in the sense of Definition 6 with respect to the outer-measure obtained by the Carthéodory extension of the length function $\ell$ defined on the ring of bounded intervals (resp. the Lebesgue measure $\lambda$ defined on a $\sigma$-algebra of subsets of $\Omega$ ).

It should also be noticed that if the set $A$ is such that $\eta(A)=0$, then for all subpartitions $P \in \Pi(A)$, $\eta_{f}(P)=0$, and thus $\int_{A} f \mathrm{~d} \eta=0$. It follows that the integral does not distinguish between functions which differ only on set of size zero. More precisely,

$$
\begin{aligned}
& \int_{A} f \mathrm{~d} \eta=\int_{A} g \mathrm{~d} \eta \text { whenever } \\
& \eta\{x \in A: f(x) \neq g(x)\}=0 .
\end{aligned}
$$

We say that a function $f$ is $\eta$-essentially equal on $A$ to another function $g$, and we write $f^{\eta} \sim g$, if $\eta\{x \in A: f(x) \neq g(x)\}=0$. It is readily seen that the relation $f^{\eta} \sim g$, is an equivalence relation on $\mathcal{I}(A, X, \eta)$. We shall denote by $I(A, \eta, X)$ the quotient space $\mathcal{I}(A, X, \eta) / \stackrel{\eta}{\sim}$.

Definition 8. For every $f: A \rightarrow X$, we define the variation of $f$ on $A$ to be

$$
\|f\|_{\Pi}:=\sup \left\{\left\|\eta_{f}(P)\right\|: P \in \Pi(A)\right\} .
$$

We say that the function $f$ is of bounded variation if $\|f\|_{\Pi}<\infty$.

The collection of all functions of bounded variation on $A$ will be denoted by $B V(A, X, \eta)$. It is clear that $\mathcal{I}(A, X, \eta) \subset B V(A, X, \eta)$ and that $f \mapsto\|f\|_{\Pi}$ defines a seminorm on $B V(A, X, \eta)$. For the particular case where $\eta(A)$ is finite, a slight modification of the proof of Theorem 3 of [5] leads to the following result.

Theorem 9. Let $\eta: 2^{\Omega} \rightarrow[0, \infty]$ be a size-function and $X$ a Banach space. Let $A \in \Sigma$ be such that $\eta(A)<\infty$. Then the function space $\mathcal{I}(A, X, \eta)$ is complete with respect to the seminorm \|\|$_{\Pi}$.

Proof. Let $n \mapsto f_{n}$ be a Cauchy sequence in $\mathcal{I}(A, X, \eta)$ with respect to the seminorm \|\|$_{\Pi}$. Fix $\varepsilon>0$, and let $N_{\varepsilon}>0$ such that for $m, n>N_{\varepsilon}$ in $\mathbb{N}$,

$$
\left\|f_{n}-f_{m}\right\|=\sup \left\{\left\|\eta_{f_{n}-f_{m}}(P)\right\|: P \in \Pi(A)\right\}<\varepsilon .
$$

In particular, if we consider the subpartition $\{(A, \omega)\} \in \Pi(A)$, then for $m, n>N_{\varepsilon}$ in $\mathbb{N}$,

$$
\left\|f_{n}(\omega)-f_{m}(\omega)\right\|<\eta(A) \varepsilon .
$$

Since $\eta(A)<\infty$, we infer that the sequence $n \mapsto f_{n}(\omega)$ is Cauchy in $X$. Since $X$ is a Banach 
space, we can define a function

$$
\omega \mapsto f(\omega)=\lim _{n \rightarrow \infty} f_{n}(\omega) .
$$

On the other hand, since $f_{n}, f_{m} \in \mathcal{I}(A, X, \eta)$, there exist $P_{n}, P_{m} \in \Pi(A)$ such that

$$
\begin{gathered}
\left\|\eta_{f_{n}}(P)-\int_{A} f_{n} \mathrm{~d} \eta\right\|<\varepsilon \text { whenever } P \succ P_{n}, \\
\left\|\eta_{f_{m}}(P)-\int_{A} f_{m} \mathrm{~d} \eta\right\|<\varepsilon \text { whenever } P \succ P_{m} .
\end{gathered}
$$

Combining these inequality with (2), it follows that for $m, n>N_{\varepsilon}$ in $\mathbb{N}$ and for every $P \succ P_{n} \vee P_{m}$, we have

$$
\begin{aligned}
& \left\|\int_{A} f_{n} \mathrm{~d} \eta-\int_{A} f_{m} \mathrm{~d} \eta\right\| \leq\left\|\eta_{f_{n}}(P)-\int_{A} f_{n} \mathrm{~d} \eta\right\|+\left\|\eta_{f_{n}-f_{m}}(P)\right\| \\
& +\left\|\eta_{f_{m}}(P)-\int_{A} f_{m} \mathrm{~d} \eta\right\|<3 \varepsilon .
\end{aligned}
$$

This proves that the sequence $n \mapsto \int_{A} f_{n} \mathrm{~d} \eta$ is Cauchy in $X$, and thus converges to say $a \in X$.

Now since for each $\omega \in A, \quad f(\omega)=\lim _{n \rightarrow \infty} f_{n}(\omega)$, there exists $N_{\omega}>N_{\varepsilon}$ such that for $m, n>N_{\omega}$ in $\mathbb{N}$,

$$
\left\|f_{n}(\omega)-f_{m}(\omega)\right\| \leq \frac{\varepsilon}{\eta(A)} .
$$

It follows that for $P=\left\{\left(I_{i}, t_{i}\right): i \in\{1, \cdots, k\}\right\} \in \Pi(A)$, and for every $m, n>\max \left\{N_{t_{i}}: i=1, \cdots, k\right\}=: N_{P}$, we have

$$
\left\|\eta_{f_{n}-f_{m}}(P)\right\| \leq \sum_{i=1}^{k} \eta\left(I_{i}\right)\left\|f_{n}\left(t_{i}\right)-f_{m}\left(t_{i}\right)\right\| \leq \varepsilon .
$$

If we let $m \rightarrow \infty$, we obtain $\left\|\eta_{f_{n}-f}(P)\right\| \leq \varepsilon$. Since $a=\lim _{m \rightarrow \infty} \int_{A} f_{m} \mathrm{~d} \eta$, there exists $N>N_{P}$ such that $\left\|\int_{A} f_{m} \mathrm{~d} \eta-a\right\|<\varepsilon$ whenever $m>N$. Thus for $n, m>N$,

$$
\begin{aligned}
\left\|\eta_{f}(P)-a\right\| \leq & \left\|\eta_{f_{n}-f}(P)\right\|+\left\|\eta_{f_{n}-f_{m}}(P)\right\| \\
& +\left\|\int_{A} f_{m} \mathrm{~d} \eta-a\right\|<3 \varepsilon .
\end{aligned}
$$

Since $\varepsilon>0$ is arbitrary, this shows that $f \in \mathcal{I}(A, X, \eta)$ and that $\int_{A} f \mathrm{~d} \eta=a$.

It goes without saying that when the $\eta$-equivalent functions taking values in a Banach space $X$ are identified, the restriction of the seminorm \|\|$_{\Pi}$ defines a norm on $I(A, X, \eta)$, and gives the space $I(A, X, \eta)$, the structure of a Banach space.

\section{Extended Lebesgue-NikoDým Theorem}

Again, we fix a (real or complex) Banach space $X$. Let $\eta: \Sigma \subset 2^{\Omega} \rightarrow[0, \infty]$ a size-function. We say that a set function $F: \Sigma \rightarrow X$ is $\eta$-absolutely continuous on $\Sigma$ if for every $\varepsilon>0$, there exists $\delta>0$ such that $\|F(A)\|<\varepsilon$ whenever $A \in \Sigma$ with $\eta(A)<\delta$.

Loosely speaking, our main result states that $\eta$-absolute continuity implies $\eta$-differentiability for additive set functions. It naturally extends the Lebesgue-Nikodým Theorem in its "full generality". Namely.

Theorem 10. Let $X$ be a Banach space, $\eta: \Sigma \rightarrow[0, \infty]$ a size-function. Assume that $F: \Sigma \rightarrow X$ an additive set function that is $\eta$-absolutely continuous on $\Sigma$. Then there exists an $f \in \mathcal{I}(\Omega, X,(\Sigma, \eta))$ such that

$$
F(A)=\int_{A} f \mathrm{~d} \eta
$$

for all $A \in \Sigma$, with $\eta(A)<\infty$.

Remark. Note that, unlike the Radon-Nikodým derivative of a vector measure, the above density function $f$ need not be measurable nor Bochner integrable. It is not even required that the scalar function $\omega \mapsto\|f(\omega)\|$ be integrable in the sense of Definition 6. Such a function $f$ will simply be called the $\eta$-derivative of the set function $F$.

Proof. In view of Proposition 3 and Proposition 7, we may assume without loss of generality that $\Sigma=2^{\Omega}$. Fix $A \in 2^{\Omega}$, with $\eta(A)<\infty$. For every subpartition $P \in \Pi(\Omega)$, consider the function defined on $\Omega$ by

$$
F_{P}(\omega)=\sum_{I \in P} \frac{1_{I}(\omega)}{\eta(I \cap A)} F(I \cap A) .
$$

Here $1_{I}$ denotes the indicator function of the set $I$. Then it is easily seen that $F_{P} \in \mathcal{I}(\Omega, \eta, X)$. The $\eta$ absolute continuity of $F$ ensures that

$$
\begin{aligned}
\int_{A} F_{P} \mathrm{~d} \eta & =\sum_{I \in P} F(I \cap A) \\
& =F\left(\bigsqcup_{I \in P} I \cap A\right) \rightarrow F(A)
\end{aligned}
$$

as $\eta\left(\bigsqcup_{I \in P} I \cap A\right) \rightarrow \eta(A)$. We claim that the net $P \mapsto F_{P}$ is Cauchy with respect to the seminorm $\|\cdot\|_{\Pi}$. Fix $\varepsilon>0$. By $\eta$-absolutely continuity of $F$, we can find $P$ and $Q$ so refined that

$$
\begin{aligned}
& \left\|F\left(\bigsqcup_{I \in P} I \cap A\right)-F\left(\bigsqcup_{J \in Q} I \cap A\right)\right\| \\
& =\left\|F\left(\bigsqcup I \cap A \backslash \bigsqcup_{J \in Q} I \cap A\right)\right\|<\frac{\varepsilon}{3} .
\end{aligned}
$$

For such $P$ and $Q$, there exists $R_{0} \in \Pi(A)$ such that for $R \succ R_{0}$,

$$
\left\|\eta_{F_{P}}(R)-\int_{A} F_{P} \mathrm{~d} \eta\right\|<\frac{\varepsilon}{3}, \text { and }\left\|\eta_{F_{Q}}(R)-\int_{A} F_{Q} \mathrm{~d} \eta\right\|<\frac{\varepsilon}{3} \text {. }
$$

It follows from (4), (5) that for $R \succ R_{0}$,

$$
\begin{aligned}
& \left\|\eta_{F_{P}}(R)-\eta_{F_{Q}}(R)\right\| \leq\left\|\eta_{F_{P}}(R)-\int_{A} F_{P} \mathrm{~d} \eta\right\| \\
& +\left\|\int_{A} F_{Q} \mathrm{~d} \eta-\eta_{F_{Q}}(R)\right\| \\
& +\left\|F\left(\bigsqcup_{I \in P} I \cap A\right)-F\left(\bigsqcup_{J \in Q} I \cap A\right)\right\| \leq \varepsilon .
\end{aligned}
$$


This proves our claim.

By Theorem 9, there exists $f \in \mathcal{I}(\Omega, X, \eta)$ such that the net $P \mapsto F_{P}$ converges to $f$ with respect to the seminorm $\|\cdot\|_{\Pi}$. Thus given $\varepsilon>0$, there exists a tagged subpartition $P_{1} \in \Pi(A)$ such that for $R \succ P_{1}$

$$
\sup \left\{\left\|\eta_{f}(R)-\eta_{F_{P}}(R)\right\|: R \in \Pi(A)\right\}<\frac{\varepsilon}{3} .
$$

On the other hand, it follows from (3) that there exists $P_{2} \in \Pi(A)$ such that for $R \succ P_{2}$

$$
\left\|\eta_{F_{P}}(R)-F(A)\right\| \leq \frac{\varepsilon}{3}
$$

Finally, by definition of the integral, there exists $P_{3} \in \Pi(A)$ such that for $R \succ P_{3}$

$$
\left\|\int_{A} f \mathrm{~d} \eta-\eta_{f}(R)\right\| \leq \frac{\varepsilon}{3} .
$$

Combining (7), (8), and (9), we have for $R \succ P_{1} \vee P_{2} \vee P_{3}$

$$
\begin{aligned}
\left\|\int_{A} f \mathrm{~d} \eta-F(A)\right\| \leq & \left\|\int_{A} f \mathrm{~d} \eta-\eta_{f}(R)\right\| \\
& +\left\|\eta_{f}(R)-\eta_{F_{P}}(R)\right\| \\
& +\left\|\eta_{F_{P}}(R)-F(A)\right\| \leq \varepsilon .
\end{aligned}
$$

The desired result follows since $\varepsilon>0$ is arbitrary chosen. The proof is complete.

As a direct corollary of our main Theorem 10, we do have the following extended version of the vector valued Radon-Nikodým Theorem.

Corollary 11. Let $\Sigma$ be a $\sigma$-algebra of a set $\Omega$ and $\mu: \Sigma \rightarrow X$ is a countably additive vector measure that is absolutely continuous with respect to a finite measure $\lambda: \Sigma \rightarrow[0, \infty)$. Then there exists an $f \in \mathcal{I}(\Omega, X, \lambda)$ (not necessarily Bochner integrable) such that

$$
\mu(A)=\int_{A} f \mathrm{~d} \lambda
$$

for all $A \in \Sigma$.

Proof. We notice that the vector measure $\mu$ is an additive set function and that the outer-measure obtained by the Carathéodory extension of the measure $\lambda$ defines a size-function on $2^{\Omega}$. The desired result is obtained by applying Theorem 10 to the set function $\mu$ and the size-function $\lambda$.

Remark. Note again that in the above Corollary 11, the density function $f$ is not a Radon-Nikodým derivative. It is also important to realize that in contrast with the classical vector valued Radon-Nikodym Theorem, the boundedness of the variation of the set function $\mu$ is not required here.

It is a well known fact that if the $\lambda$-density function $f$ of a vector measure $\mu$ exists and is Bochner integrable with respect to $\lambda$, then $\mu$ must have bounded variation and its variation is given by

$$
|\mu|(A)=\int_{A}\|f\| \mathrm{d} \lambda
$$

for every $A \in \Sigma$.

Recall that a Banach space is said to have the RadonNikodým Property if every vector measure $\mu: \Sigma \rightarrow X$ of bounded variation that is absolutely continuous with respect to a finite measure $\lambda: \Sigma \rightarrow[0, \infty)$ has a Radon-Nikodým derivative. We end this section with the following immediate corollary.

Corollary 12. A Banach space $X$ has the RadonNikodým Property if and only if for every $\sigma$-algebra $\Sigma$ of a set $\Omega$, for every vector measure $\mu: \Sigma \rightarrow X$ of bounded variation that is absolutely continuous with respect to a finite measure $\lambda: \Sigma \rightarrow[0, \infty)$, the $\lambda$-derivative of $\mu$ is a Radon-Nikodým derivative.

\section{Extended Fundamental Theorem of Calculus}

In this section, we give conditions under which a given vector valued function (not necessarily measurable) is the derivative of a given finitely additive set-function. Our result can be compared to that of Lu and Pee in [6].

It clearly follows from our definition of the integral that $f \in \mathcal{I}(\Omega, X, \eta)$ if and only if $f \in \mathcal{I}(A, X, \eta)$ for every subsets $A$ of $\Omega$. This gives rise to a set function $F: \Sigma \rightarrow X: F(A)=\int_{A} f \mathrm{~d} \eta$ for all $A \in \Sigma \subset 2^{\Omega}$. It immediately follows from the properties of the integral that such a set function is finitely additive. Such a set function $F$ is called the $\eta$-indefinite integral of the function $f$. Of course, an integrable function $f$ is always the $\eta$-derivative of its $\eta$-indefinite integral.

The next result gives a necessary and sufficient condition under which an additive set function $F$ defined on $2^{\Omega}$ is the $\eta$-indefinite integral of an integrable function $f: \Omega \rightarrow X$. In what follows the size-function $\eta$ is considered to be finite, that is, $\eta(\Omega)<\infty$.

Theorem 13. Let $\Omega$ be a nonempty set and $\eta: 2^{\Omega}$ $\rightarrow[0, \infty)$ be a finite size-function. Then the following statements are equivalent for an additive set function $F: 2^{\Omega} \rightarrow X$ and a function $f: \Omega \rightarrow X$.

1) $f \in \mathcal{I}(\Omega, X, \eta)$ and $F$ is the $\eta$-indefinite integral of $f$.

2) For every $\varepsilon>0$, there exists $P_{\varepsilon} \in \Pi(\Omega)$ consisting of elements of

$$
\Gamma_{\varepsilon}:=\{(I, \omega) \in \Pi(\Omega):\|F(I)-\eta(I) f(\omega)\| \geq \eta(I) \varepsilon\}
$$

such that for every $P \succ P_{\varepsilon}$,

$$
\sum_{I_{t} \in P \cap \Gamma_{\varepsilon}}\left\|F\left(I_{t}\right)\right\|<\varepsilon \text { and } \sum_{I_{t} \in P \cap \Gamma_{\varepsilon}} \eta\left(I_{t}\right)\|f(t)\|<\varepsilon .
$$

Proof. For the necessity, fix $\varepsilon>0$ and let $P_{\varepsilon} \in \Pi(\Omega)$ as in 2. Then for $P \succ P_{\varepsilon}$ 


$$
\begin{aligned}
& \left\|F(\Omega)-\eta_{f}(P)\right\| \leq\left\|F(\Omega)-\eta_{f}(P)\right\| \\
& \leq \sum_{I_{t} \in P}\left\|F\left(I_{i}\right)-\eta\left(I_{i}\right) f(t)\right\| \\
& \leq \sum_{I_{t} \in P \backslash \Gamma_{\varepsilon}}\left\|F\left(I_{i}\right)-\eta\left(I_{i}\right) f(t)\right\| \\
& +\sum_{I_{t} \in P \cap \Gamma_{\varepsilon}}\left\|F\left(I_{i}\right)-\eta\left(I_{i}\right) f(t)\right\| \\
& \leq \varepsilon \sum_{I_{t} \in P \backslash \Gamma_{\varepsilon}} \eta\left(I_{t}\right)+\sum_{I_{t} \in P \cap \Gamma_{\varepsilon}}\left\|F\left(I_{t}\right)\right\| \\
& +\sum_{I_{t} \in P \cap \Gamma_{\varepsilon}} \eta\left(I_{t}\right)\|f(t)\| \\
& \leq \varepsilon(\eta(\Omega)+2) .
\end{aligned}
$$

Since $\varepsilon>0$ is arbitrary, this shows that

$$
f \in \mathcal{I}(\Omega, X, \eta) \text { and } \int_{\Omega} f \mathrm{~d} \eta=F(\Omega) .
$$

Conversely, assume that $f \in \mathcal{I}(\Omega, X, \eta)$. Let

$$
E_{k}=\{\omega \in \Omega: k-1 \leq f(\omega)<k\} .
$$

Given $\varepsilon>0$ there exists a $\eta$-subpartition $P_{k, \varepsilon} \in \Pi(\Omega)$ such that for every $P \succ P_{k, \varepsilon}$,

$$
\sum_{I_{t} \in P}\left\|F\left(I_{t}\right)-\eta\left(I_{t}\right) f(t)\right\| \leq \frac{\varepsilon^{2}}{k 2^{k+1}} .
$$

For each $n \in \mathbb{N}$, let

$$
f_{n}(\omega)= \begin{cases}f(\omega) & \text { if } \omega \in \bigcup_{k=1}^{n} E_{k} \\ 0 & \text { otherwise. }\end{cases}
$$

Then for $P \succ \vee_{k=1}^{n} P_{k, \varepsilon}$, we have

$$
\begin{aligned}
& \sum_{I_{t} \in P \cap \Gamma_{\varepsilon}} \eta\left(I_{t}\right)\left\|f_{n}(t)\right\| \leq \sum_{I_{t} \in P \cap \Gamma_{\varepsilon}} \eta\left(I_{t}\right) k \\
\leq & \sum_{I_{t} \in P \cap \Gamma_{\varepsilon}} \frac{k}{\varepsilon}\left\|F\left(I_{t}\right)-\eta\left(I_{t}\right) f(t)\right\| \leq \frac{\varepsilon}{2} .
\end{aligned}
$$

On taking the limit as $n \rightarrow \infty$, we infer that

$$
\sum_{I_{t} \in P \cap \Gamma_{\varepsilon}} \eta\left(I_{t}\right)\|f(t)\| \leq \frac{\varepsilon}{2} .
$$

It then follows that

$$
\begin{aligned}
\sum_{I_{t} \in P \cap \Gamma_{\varepsilon}}\left\|F\left(I_{t}\right)\right\| \leq & \sum_{I_{t} \in P \cap \Gamma_{\varepsilon}}\left\|F\left(I_{t}\right)-\eta\left(I_{t}\right) f(t)\right\| \\
& +\sum_{I_{t} \in P \cap \Gamma_{\varepsilon}} \eta\left(I_{t}\right)\|f(t)\| \leq \frac{\varepsilon}{2}+\frac{\varepsilon}{2}=\varepsilon .
\end{aligned}
$$

The proof is complete.

Let now assume that $\Omega$ is a topological space. We say that a size-function $\eta: 2^{\Omega} \rightarrow[0, \infty]$ is regular if it is non-zero on open sets of $\Omega$. For a fixed $\omega \in \Omega$, let $\theta_{\Omega}(\omega)$ the set of all neighborhoods of $\omega$. Then $\theta_{\Omega}(\omega)$ is directed by inclusion $\subset$. We introduce the following definition.

Definition 14. Let $\Omega$ be a topological space and $\eta: 2^{\Omega} \rightarrow[0, \infty]$ a regular size-function. Let $\Sigma \subset 2^{\Omega}$ contain the topology of $\Omega$. A set function $F: \Sigma \rightarrow X$ is said to be $\eta$-differentiable at a point $\omega \in \Omega$, if $\lim _{\left(\theta_{\Omega}(\omega), \subset\right)} \frac{F}{\eta}$ exists in $X$.

In other words, $F: \Sigma \rightarrow X$ is $\eta$-differentiable at a point $\omega \in \Omega$, if there exists a vector $f(\omega) \in X$ such that for every $\varepsilon>0$, there exists open neighborhood $U_{\varepsilon}(\omega) \in \theta_{\Omega}(\omega)$, such that for every $U \in \theta_{\Omega}(\omega)$, $U \subset U_{\varepsilon}(\omega)$,

$$
\|F(U)-\eta(U) f(\omega)\|<\eta(U) \varepsilon .
$$

We call the set $\Delta_{F}=\left\{\omega \in \Omega: \lim _{\left(\theta_{\Omega}(\omega), \subset\right)} \frac{F}{\eta}\right.$ exists $\}$ the domain of differentiability of $F$. By the uniqueness of net limit, the correspondence

$$
\omega \mapsto \lim _{\left(\theta_{\Omega}(\omega), \subset\right)} \frac{F}{\eta}
$$

defines a function on $\Delta_{F}$ denoted by $F^{\prime}$ and called the $\eta$-derivative of $F$.

Now assume that $\Omega$ is compact. Let $\Sigma \subset 2^{\Omega}$ containing the topology of $\Omega$, and $F: 2^{\Omega} \rightarrow X$ an additive set function such that $\Delta_{F}=\Omega \backslash E$, with $\eta(E)=0$. Given $\varepsilon>0$, let $U_{\varepsilon}$ be an open set such that $E \subset U_{\varepsilon}$ and $\eta\left(U_{\varepsilon}\right)<\varepsilon$. On the other hand, for every $\omega \in \Delta_{F}$, there exists an open neighborhood $U_{\varepsilon}(\omega) \in \theta_{\Omega}(\omega)$ such that for every $U \subset U_{\varepsilon}(\omega), U \in \theta_{\Omega}(\omega)$,

$$
\|F(U)-\eta(U) f(\omega)\|<\eta(U) \varepsilon .
$$

Clearly, $\left\{U_{\varepsilon}(\omega): \omega \in \Delta_{F}\right\} \bigcup\left\{U_{\varepsilon}\right\}$ is an open cover of $\Omega$. By compactness, a finite subcover exists, say

$$
\left\{U_{\varepsilon}\left(\omega_{i}\right): \omega_{i} \in \Delta_{F}, i=1, \cdots, n\right\} \cup\left\{U_{\varepsilon}\right\} .
$$

Let $P_{\varepsilon} \in \Pi(\Omega)$ such that for

$$
\left\|P_{\varepsilon}\right\| \leq \min \left\{\eta\left(U_{\varepsilon}\right), \eta\left(U_{\varepsilon}\left(\omega_{i}\right)\right): i=1, \cdots, n\right\} .
$$

Then the set $P_{\varepsilon} \cap \Gamma_{\varepsilon}=\varnothing$, and 1. of Theorem 13 is satisfied by $F$ and any arbitrary extension of $F^{\prime}$ to $\Omega$. It follows that since the size-function $\eta$ is of bounded variation, then any such extension is an element of $\mathcal{I}(\Omega,(\Sigma, \eta), X)$ and $F$ is its $\eta$-indefinite integral. Hence we have the following result which can be seen as a generalization of the Fundamental Theorem of Calculus.

Theorem 15. Let $\Omega$ be a compact topological space, $\Sigma \subset 2^{\Omega}$ containing the topology of $\Omega$ and $\eta: 2^{\Omega} \rightarrow[0, \infty]$ be a finite regular size-function. Then every additive set function $F: \Sigma \rightarrow X$ with domain of differentiability 
$\Delta_{F}=\Omega \backslash E$, with $\eta(E)=0$, is the $\eta$-indefinite integral of any arbitrary extension of its $\eta$-derivative.

\section{REFERENCES}

[1] M. A. Robdera, "Unified Approach to Vector Valued Integration," International Journal of Functional Analysis, Operator Theory and Application, Vol. 5, No. 2, 2013, pp. 119-139.

[2] S. Bochner, "Additive Set Functions on Groups," Annals of Mathematics, Second Series, Vol. 40, No. 4, 1939, pp. 769-799.

[3] J. Diestel and J. J. Uhl Jr., "Vector Measures," American
Mathematical Society, Providence, R.I., 1977.

[4] E. J. McShane, "Partial Orderings and Moore-Smith Limits," American Mathematical Monthly, Vol. 59, No. 1, 1952, pp. 1-11. http://dx.doi.org/10.2307/2307181

[5] M. A. Robdera, "On Strong and Weak Integrability of Vector Valued Functions," International Journal of Functional Analysis, Operator Theory and Application, Vol. 5, No. 1, 2013, pp. 63-81.

[6] J. T. Lu and P. Y. Pee, "The Primitive of a Henstock Integrable Functions in Euclidean Space," Bulletin of the London Mathematical Society, Vol. 31, No. 2, 1999, pp. 173-180. http://dx.doi.org/10.1112/S0024609398005347 